ACCEPTED MANUSCRIPT

\title{
Time-resolved laser-induced fluorescence diagnostics for electric propulsion and their application to breathing mode dynamics
}

To cite this article before publication: Christopher Valentine Young et al 2018 Plasma Sources Sci. Technol. in press https://doi.org/10.1088/1361$\underline{6595 / \text { aade42 }}$

\author{
Manuscript version: Accepted Manuscript \\ Accepted Manuscript is "the version of the article accepted for publication including all changes made as a result of the peer review process, \\ and which may also include the addition to the article by IOP Publishing of a header, an article ID, a cover sheet and/or an 'Accepted \\ Manuscript' watermark, but excluding any other editing, typesetting or other changes made by IOP Publishing and/or its licensors" \\ This Accepted Manuscript is @ 2018 IOP Publishing Ltd.
}

During the embargo period (the 12 month period from the publication of the Version of Record of this article), the Accepted Manuscript is fully protected by copyright and cannot be reused or reposted elsewhere.

As the Version of Record of this article is going to be / has been published on a subscription basis, this Accepted Manuscript is available for reuse under a CC BY-NC-ND 3.0 licence after the 12 month embargo period.

After the embargo period, everyone is permitted to use copy and redistribute this article for non-commercial purposes only, provided that they adhere to all the terms of the licence https://creativecommons.org/licences/by-nc-nd/3.0

Although reasonable endeavours have been taken to obtain all necessary permissions from third parties to include their copyrighted content within this article, their full citation and copyright line may not be present in this Accepted Manuscript version. Before using any content from this article, please refer to the Version of Record on IOPscience once published for full citation and copyright details, as permissions will likely be required. All third party content is fully copyright protected, unless specifically stated otherwise in the figure caption in the Version of Record.

View the article online for updates and enhancements. 


\title{
Time-resolved laser-induced fluorescence diagnostics for electric propulsion and their application to breathing mode dynamics
}

\author{
C V Young \\ Stanford Plasma Physics Laboratory, Stanford University, Stanford, CA 94305, USA \\ Presently: Lawrence Livermore National Laboratory, 7000 East Ave., Livermore, CA \\ 94550, USA \\ E-mail: cvyoung@alumni.stanford.edu

\section{A Lucca Fabris} \\ Surrey Space Centre, University of Surrey, Guildford GU2 7XH, UK
}

N A MacDonald-Tenenbaum, W A Hargus

Air Force Research Laboratory, Edwards Air Force Base, CA 93524, USA

\section{A Cappelli}

Stanford Plasma Physics Laboratory, Stanford University, Stanford, CA 94305, USA

\begin{abstract}
.
Several techniques have been déveloped recently for performing time-resolved laser-induced fluorescence (LIF) measurements in oscillating plasmas. One of the primary applications is characterizing plasma fluctuations in devices like Hall thrusters used for space propulsion. Optical measurements such as LIF are nonintrusive and can resolve properties like ion velocity distribution functions with high resolution in velocity and physical space. The goals of this paper are twofold. First, the various methods proposed by the community for introducing time resolution into the standard LIF measurement of electric propulsion devices are reviewed and compared in detail. Second, one of the methods, the sample-hold technique, is enhanced by parallelizing the measurement hardware into several signal processing channels that vastly increases the data acquisition rate. The new system is applied to study the dynamics of ionization and ion acceleration in a commercial BHT-600 Hall thruster undergoing unforced breathing mode oscillations in the $44-49 \mathrm{kHz}$ range. A very detailed experimental picture of the common breathing mode ionization instability emerges, in close agreement with established theory and numerical simulations.
\end{abstract}

Keywords: time-resolved laser-induced fluorescence, plasma diagnostics, electric propulsion, Hall thruster, breathing mode, plasma oscillation. Submitted to: Plasma Sources Sci. Technol. 


\section{Introduction}

Electric propulsion (EP) systems are presently used for many types of space missions. In such devices, propellant is accelerated to high exhaust velocities via electrical heating or electromagnetic forces (or both) [1], far outstripping those achievable by the thermodynamic expansion of combustion gases used with chemical propulsion [2]. When undergoing a given orbital maneuver (defined by a velocity change $\Delta V$ ), this higher exhaust velocity enables savings in propellant mass, an attractive feature of EP. Such systems also produce a low level of thrust, constrained by the limited available electrical power, that can be sufficient in some mission scenarios (drag compensation, station keeping) but represents a major drawback in other scenarios (e.g. orbit transfer with limited transfer time).

Some electric propulsion devices developed over the past 60 years $[3,4]$ have matured into established technologies, accumulating extensive spaceflight heritage, including gridded ion engines (GIE) and Hall effect thrusters (HET), while more recent concepts remain at a low technology readiness level. Hall thrusters $[5,6]$ are one of the workhorses of modern electric propulsion that offer an attractive option for low thrust, high specific impulse mission requirements such as satellite station keeping, orbit raising and deep space travel. As representative examples, the European Space Agency SMART-1 spacecraft [7] used a Hall thruster to move from a geostationary transfer to a polar moon orbit, and several commercial telecommunications satellites leverage Hall thrusters for geostationary orbit north-south station keeping [8]. In the latter case, recent developments have now enabled "all-electric" platforms that utilize Hall thrusters also for orbit raising [9].

At their core, Hall thrusters are essentially a plasma discharge sustained by a combination of electric and magnetic fields that efficiently ionize propellant (usually xenon) and accelerate the resulting ions to high velocity. Neutral propellant atoms are injected around the anode, located at the closed end of the discharge channel, and diffuse downstream towards the thruster exit plane. Along the way, neutral atoms are ionized via electron-impact collisions with primary electrons provided by an external source (cathode) and secondary electrons from previous ionization events. In the standard annular configuration [5], a primarily radial magnetic field is applied in a localized region near the exit plane to reduce the axial electron mobility and form a transport barrier between the external cathode and the anode. A primarily axial electric field arises from this enhanced electron resistivity, and an $\mathbf{E} \times \mathbf{B}$ electron drift is established in the azimuthal direction around the annular channel. Ions produced in the discharge channel are mostly unmagnetized due to their high mass, and accelerate electrostatically under the influence of the axial electric field. The typical electrostatic potential difference between the anode and cathode is $\sim 300 \mathrm{~V}$. The spatial potential distribution is shaped by the local plasma resistivity, resulting in a strong potential drop near the thruster exit plane. The ejected ion beam is neutralized by electrons emitted from the external electron source, preserving the overall charge balance of the spacecraft. 
Despite decades of study, many fundamental physical processes that determine the overall operation of Hall thrusters are still not fully understood and are the subject of ongoing research. Basic information such as where propellant is being ionized, the precise location and form of the accelerating potential [10], the speed and direction in which ions are ejected [11, 12], and how electrons are transported across the predominantly radial magnetic field lines of the channel and near field plume $[13,14]$ are not easily modeled or calculated, and detailed measurements are needed to calibrate existing analytical or numerical models. In addition, a plethora of instabilities and oscillations over a broad frequency range $(1 \mathrm{kHz}$ to $60 \mathrm{MHz})[3,15]$ can arise in certain operating conditions, further complicating the physical picture. In some cases, their presence might deteriorate the operating efficiency and lifetime of the device. One of the strongest oscillatory regimes is a type of ionization instability called the breathing mode $[16,17]$, associated with alternating cycles of efficient propellant ionization, expulsion of ions, and replenishment of neutrals. At a macroscopic level, this process gives rise to intense quasi-periodic discharge current oscillations. This interplay between avalanche ionization and neutral gas transport has been modeled as a predator-prey type relationship [18, 19] between the neutral propellant and ions released behind a traveling ionization front. A first order linear analysis by Fife [18] shows the dependence of the breathing mode frequency $f_{\mathrm{b}}$ on the (time-ayeraged) ion and neutral velocities, $v_{\mathrm{i}}$ and $v_{\mathrm{n}}$ respectively, and the characteristic length of the ionization zone, $L$ :

$$
f_{\mathrm{b}}=\frac{1}{2 \pi} \frac{\sqrt{v_{\mathrm{i}} v_{\mathrm{n}}}}{L}
$$

This relation yields fundamental harmonics in the $10-50 \mathrm{kHz}$ range, in agreement with experimental observations. Subsequent enhancements have been made to this straightforward description $[17,20]$, highlighting the complex interplay between the dynamics of the ionization front, the evolution of the potential topology established in the channel, and the acceleration of ions during breathing mode oscillations.

Experiments using both physical and optical probes [21, 22, 23] have indirectly verified this plasma physics picture. There is evidence, however, that the presence of physical probes near regions of propellant ionization and acceleration can perturb the operation of the discharge [24]. So, optical diagnostics, including passive measurements like emission studies $[25,26]$ and active measurements like laser-induced fluorescence (LIF) $[11,12]$, are preferable for experimentally investigating breathing mode dynamics. Only recently have the requisite time-resolved optical diagnostics become available for a close, noninvasive examination of the interior of the discharge channel with high spatial and temporal resolution. Previous time-resolved LIF studies of Hall thrusters $[27,28,29,30]$ have uncovered temporal modulations in the ion velocity and probed metastable ion density due to the breathing mode. (Note that most LIF experiments probe the metastable xenon ion level, which is linked to the ground state or absolute xenon ion density through a complex network of collisional and radiative processes [31, 32].) Many such studies used methods for time resolution that only permitted measurement in a few locations in the Hall thruster plume or at a restricted 
set of operating conditions to complete experiments in a reasonable amount of time.

This paper presents a brief review of the development of LIF for Hall thrusters, and, in particular, the more recent extension of LIF to study thruster time-dependent xenon ion velocity fields. We illustrate the application of a particular time-resolved continuous-wave LIF technique, the sample-hold method, to several spatial locations in the channel of a commercial BHT-600 Hall thruster operating in a strong breathing mode condition. The various phases of breathing mode physics are now able to be recovered experimentally in much higher detail than previously available and are discussed in the context of the preceding literature. Ion velocity distributions, obtained throughout the channel and near-field plume, are resolved over the oscillation period, tracking the progression of the electric field and propellant ionization front in both space and time. This study produces a complete breathing mode dataset for model validation and calibration, bringing new insight into the complex physics of the breathing mode.

The paper is organized as follows. Section 2 discusses the LIF diagnostic as historically applied to plasma propulsion devices and presents a short review of the various approaches for time-resolved LIF recently deployed by the community $[33,34,27,35,36]$. Section 3 describes the commercial BHT-600 Hall thruster and discusses the emerging physical picture of breathing mode dynamics derived from a large dataset of time-resolved LIF data. Concluding remarks appear in Section 4.

\section{Laser-Induced Fluorescence in Electric Propulsion}

\subsection{Brief History of Xe LIF}

The application of laser-induced fluorescence (LIF) measurements to Hall thrusters grew out of the successful development of laser fluorescence velocimetry of hydrogen in arcjet thrusters [37, 38, 39, 40, 41, 42, 43]. Initial studies in Hall thrusters focused on probing the velocity of the excited xenon neutrals and ions using argon-ion pumped ring-dye and early solid state continuous-wave lasers [44, 45, 46, 47, 48]. Transition selection was critical, as diode lasers werè becoming readily available near the infrared-visible boundary of the spectrum.

In neutrals (Xe I), favorable results were obtained using the $6 s[3 / 2]_{2}^{\mathrm{o}}-6 p[3 / 2]_{2}$ transition at $823.2 \mathrm{~nm}[45,46]$, the lower level of which is metastable. For singlyionized (Xe II) ions, first results were obtained using the $5 d[4]_{7 / 2}-6 p[3]_{5 / 2}^{0}$ transition at $834.7 \mathrm{~nm}$ [44]. In both cases, the shift of the center of gravity of the spectral absorption feature afforded a relatively straightforward measurement of the mean velocity. However, the narrow linewidth of the diode lasers and narrow absorption features due to the relatively high atomic weight of xenon required accounting for the complex isotopic and hyperfine structure of the transitions to extract velocity distributions. Scant data was available for the $834.7 \mathrm{~nm}$ ion line's optical constants.

The $5 d[3]_{7 / 2}-6 p[2]_{5 / 2}^{0}$ Xe II visible absorption transition at $605.1 \mathrm{~nm}$, also originating from a metastable level, was identified and studied as a suitable candidate 
transition for Hall thruster ion velocimetry [45, 46]. The optical constants for this transition were documented, and the ion velocity distributions could be deconvolved from the measured excitation lineshapes [49]. However, at the time, this transition was only accessible with more cumbersome argon-ion pumped ring-dye lasers. Attempts to use velocity distributions extracted from LIF measurements of the $605.1 \mathrm{~nm}$ transition to determine the optical constants of the $834.7 \mathrm{~nm}$ transition encountered challenges [50].

Despite the lack of spectroscopic constants preventing precise lineshape deconvolution, the $834.7 \mathrm{~nm}$ transition has remained the transition of choice for studying the ion dynamics in Hall thrusters [11, 51, 52, 53, 54, 55], and providing at least a firstorder description of the ion velocity distributions [12]. Because of the relatively strong signal to background, the $834.7 \mathrm{~nm}$ transition was also the candidate transition for the development of the first time-synchronized LIF studies on xenon cusped-field thrusters $[33,56]$. It was also subsequently used in the first demonstration of the time-resolved sample-hold diagnostic on Hall thrusters [57, 30], which is described in further detail below.

\subsection{LIF Experimental Setup}

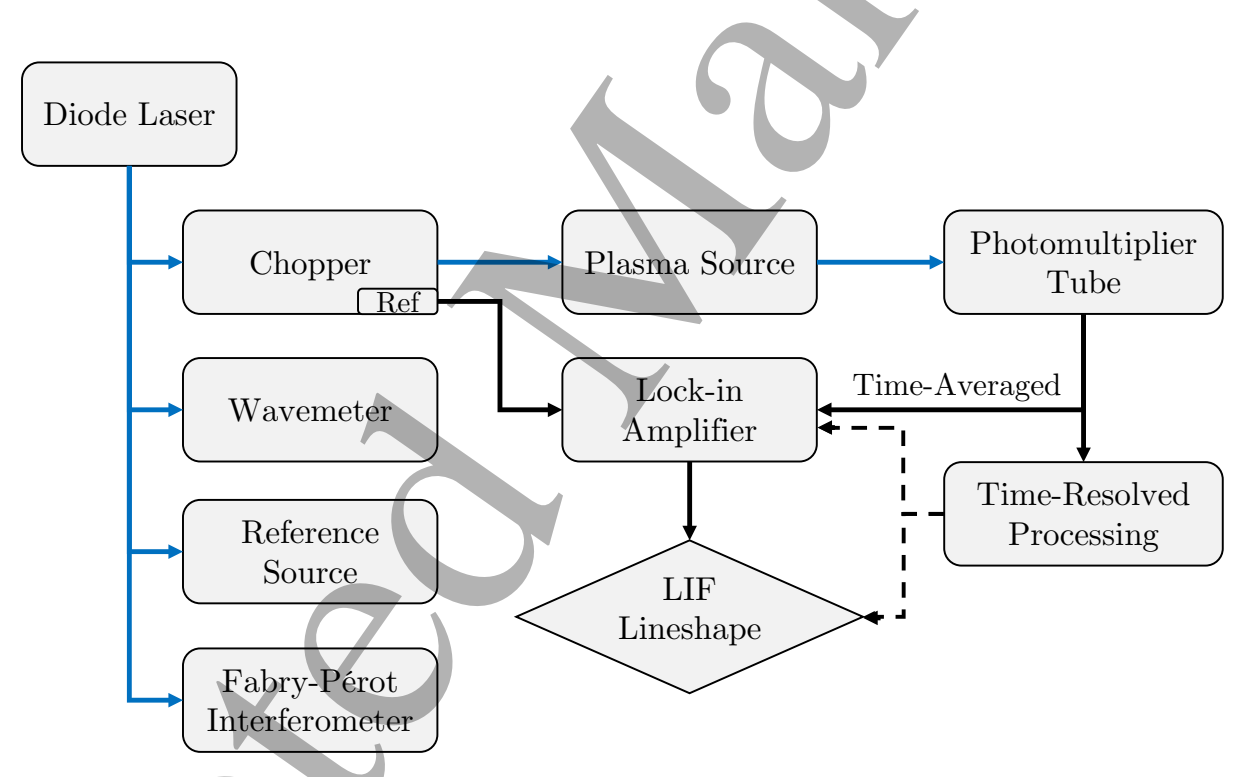

Figure 1. Simplified block diagram of a typical LIF diagnostic. Photons are denoted in blue, electrical signals in black. In time-averaged LIF, the photomultiplier (PMT) signal feeds directly to a lock-in amplifier for homodyne detection of the weak LIF signal. For time-resolved LIF, additional processing of the PMT signal may occur before the lock-in, or different electronics may be employed.

This section provides a brief overview of a typical laser-induced fluorescence experimental setup for use with electric propulsion systems, illustrated in the block diagram of Fig. 1. Much further detail may be found elsewhere [58, 59, 12, 60]. For the standard heavy atom propellants used in electric propulsion (krypton, xenon), typical valence electronic transition lineshapes are a few $\mathrm{GHz}$ in width. Continuous wave $(\mathrm{CW})$ 
lasers, with linewidths of order $\sim 50 \mathrm{kHz}$, afford much higher spectral resolution than pulsed lasers for use with these lines [33]. Finely tunable CW diode lasers are easy to operate and can be custom matched to the target transition, making them an attractive option for modern LIF diagnostics of EP systems. Tapered amplifiers can also be employed to boost laser power [30].

A tabletop wavemeter provides a visual reference of the laser frequency as it scans across the target transition. A precise measurement of laser frequency is made with a Fabry-Pérot interferometer, which produces fixed frequency markers at intervals of the free spectral range (FSR) (typically $0.3-1.5 \mathrm{GHz}$ ), and an absolute reference that must be obtained from a stationary source. One convenient choice is a hollow cathode discharge, where the zero-velocity frequency reference of the target LIF transition (or a nearby line of sufficient signal strength) can be obtained either from an absorption trace [56] or an optogalvanic measurement [30] (requiring a separate optical chopper plus lock-in amplifier system for homodyne detection of the optogalvanic signal). This information enables a highly accurate reconstruction of the instantaneous laser wavelength over time, allowing for the particle velocity distribution function $(v)$ to be inferred from the observed Doppler shift of the target lineshape $(\Delta \nu)$ away from stationary line center $\left(\nu_{0}\right)$ using the equation:

$$
v=\frac{\Delta \nu}{\nu_{0}} c
$$

where $c$ is the speed of light.

The bulk of the laser power is directed through an optical chopper (generally operating at $\sim 1-2 \mathrm{kHz}$ ) to amplitude modulate the beam, and thus the resulting LIF signal, for phase-sensitive detection with a lock-in amplifier. One particular challenge for LIF with EP is delivering the beam through the harsh plasma environment inside the test chamber and recovering the LIF photons. Systems have successfully used both optical fibers and free-space laser propagation for this task, although any optical components and windows subject to coating by sputtered material or bombardment by high energy ions released from the EP device will have a finite usable lifetime. A photomultiplier tube (PMT) with appropriate spectral filters narrows the collection range to just near the target transition, mitigating the bright broadband background emission from the plasma source. If the PMT signal is directed straight to the lock-in amplifier, a timeaveraged measurement of the LIF lineshape is obtained via phase-sensitive homodyne detection. For time resolution, additional processing of the raw PMT signal must be completed before the lock-in, or different electronics are employed altogether. Various techniques for accomplishing this are described in the following section.

\subsection{Methods for Time-Resolution with LIF}

Several techniques for obtaining time resolution in continuous wave laser-induced fluorescence studies of continuously operating plasma propulsion devices have been proposed in the literature over the past several years and are reviewed in this section. 
(Note that additional time-resolved LIF studies using pulsed discharges have also been conducted $[61,62,63]$.) All of the methods have advantages and disadvantages that make them more or less applicable in any given situation. One will notice that most techniques achieve high resolution in one aspect of the velocity distribution functions (VDFs) under measurement - either time (VDF evolution) or laser wavelength/velocity space (smoothness of each VDF) — and requires repeating the measurement process several times to build up a much lower resolution in the other variable. All but one method (transfer function averaging) requires quasi-periodic (drifting) or strictly periodic (driven) oscillations to be present in the plasma source in order to provide a time reference for obtaining the time-resolved LIF lineshape. Table 1 summarizes these qualities for each of the six methods discussed below.

Table 1. A comparison of the various methods proposed for time-resolved laserinduced fluorescence in the electric propulsion community. Each method exhibits high resolution in either time or laser frequency/VDF velocity space $(\nu, v)$, and requires repeated measurements to build up much lower resolution in the other variable.

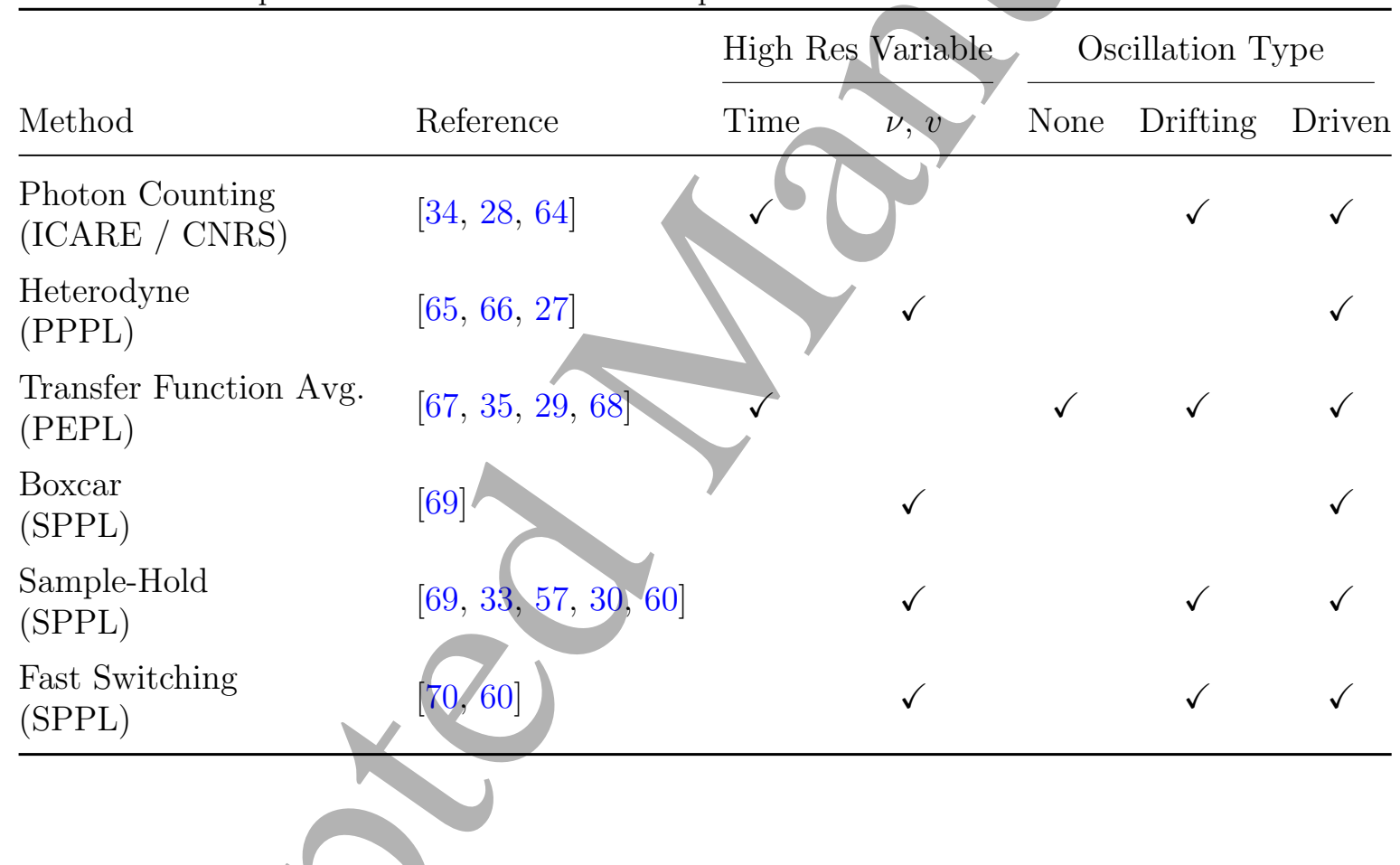

2.3.1. Photon Counting Method Developed by ICARE at CNRS Orléans, France, the photon counting method [34, 28,64] consists of a series of $100 \mathrm{~ns}$ time bins that acquire data when triggered by a signal that is synchronized with the periodic oscillation. Detected photons are distinguished from background noise with a fast amplifier and discriminator module that produces one TTL pulse per detection event. Events are tallied and either added to the running totals in each time bin when the mechanically chopped lasser beam is illuminating the discharge (fluorescence plus background), or subtracted from the running totals when the beam is blocked (just background). After $10^{5}-10^{6}$ cycles at one fixed laser frequency, sufficient signal-to-noise is achieved and the 
process is repeated at several laser frequencies to build up the time evolution of the LIF lineshape.

Since the time bins are created relative to an external trigger that is not necessarily dependent on the frequency of the discharge oscillation, the photon counting method is applicable to both natural and driven oscillating systems. However, the researchers chose to drive the discharge when applying this method to Hall thruster diagnostics to ensure a stable frequency regime. One technique, periodically extinguishing the discharge at $2.5 \mathrm{kHz}$ with a fast switch on the anode power supply, was shown to interfere with normal thruster operation [28]. The authors assert that a second approach, modulating the cathode keeper voltage near the $17 \mathrm{kHz}$ natural breathing mode, perturbs the discharge much less [64]. This method has been applied to xenon ion LIF at a few locations in the plume and channel of various thrusters $(200 \mathrm{~W}, 1.5 \mathrm{~kW}, 5 \mathrm{~kW})$.

2.3.2. Heterodyne Method The heterodyne method for time-resolved LIF was developed by researchers at the Princeton Plasma Physics Laboratory [65, 66, 27] and applied to measuring xenon ion velocity distribution functions (IVDFs) in the Cylindrical Hall Thruster (CHT) [71]. A sinusoidal modulation of 3-30 V is externally applied on top of the $220 \mathrm{~V}$ anode potential at a given frequency $\omega_{\mathrm{D}}$. The technique then assumes a decomposition of the ion IVDF $f(t, \mathbf{x}, \mathbf{v})$ into harmonics of $\omega_{\mathrm{D}}[27]$ :

$$
f(t, \mathbf{x}, \mathbf{v})=f^{0}(\mathbf{x}, \mathbf{v})+\mathcal{R} e\left\{\sum_{n>0} f^{n}(\mathbf{x}, \mathbf{v}) \exp \left[-i n \omega_{\mathrm{D}} t+i \theta_{n}(\mathbf{x}, \mathbf{v})\right]\right\}(3)
$$

where $\theta_{n}$ is the phase shift associated with each harmonic $n$. The zeroth order component $f^{0}(\mathbf{x}, \mathbf{v})$ (which is not the same as the time-averaged IVDF) is obtained from the lock-in by mixing the PMT signal with an internal reférence at the laser chopping frequency $\omega_{\mathrm{L}}$, as usual. For the higher harmonies $f^{n}(\mathbf{x}, \mathbf{v})$, the PMT is mixed with internal references at: $\omega_{\mathrm{L}} \pm n \omega_{\mathrm{D}}$, the process from which the method derives its name.

The authors found that at one location in the thruster plume, the contributions from the second order component $f^{2}$ and above $(n \geq 2)$ were negligible. A comparison of time-averaged traces with DC voltage applied to the anode near the extremes of the AC modulation (207 V and $233 \mathrm{~V}$ ) showed how the increased voltage shifted the IVDF to higher velocities; thus, the authors expected the AC anode potential modulation to induce a "sloshing" of the IVDF in the axial direction and constructed a model demonstrating this effect. The heterodyne method achieves high resolution in velocity space with the use of a scanning tunable diode laser, and the time resolution is restricted by the number of harmonics able to be resolved. Since parallel acquisition of harmonics is possible, with separate lock-ins tuned to different mixing frequencies $\omega_{\mathrm{L}} \pm n \omega_{\mathrm{D}}$, the overall measurement time could be reduced relative to the photon counting and other methods. However, depending on how sharply the IVDF changes in time and how many harmonics are resolvable, this method could suffer a loss of accuracy in distinguishing the true form of the IVDF evolution. 
2.3.3. Transfer Function Averaging Method Developed by the Plasmadynamics and Electric Propulsion Laboratory at the University of Michigan, the transfer function averaging method for time-resolved LIF is a computationally intensive approach that may be applied to systems exhibiting natural or driven oscillations, in addition to more randomized transition regions where no clear coherent oscillation is present $[67,35,29,68]$. The method attempts to track approximate real-time shifts in the IVDF on the time scale of discharge current perturbations by defining an average transfer function in the frequency domain $\langle H[k]\rangle$ that describes the output fluorescence signal $\tilde{F}[k]$ at a single wavelength for a given input discharge current trace $\tilde{I}_{\mathrm{D}}[k]$ :

$$
\tilde{F}[k]=\langle H[k]\rangle \tilde{I}_{\mathrm{D}}[k]
$$

where $[k]$ is the discrete vector index for the quantities defined in the frequency domain. The time evolution of the full IVDF is then constructed by feeding the same characteristic current trace into all of the calculated transfer functions. The technique obtains high time resolution and must be applied repeatedly for each laser wavelength to build up the IVDF. Discharge current and PMT signals are digitized at $20 \mathrm{MHz}$ for 60 seconds at each wavelength.

The transfer function method uses an acousto-optic modulator to chop the exciting laser beam at $1 \mathrm{MHz}$ in a region of low spectral noise density. The PMT signal enters a $0.9-1.1 \mathrm{MHz}$ bandpass filter before digitization and demodulation from the $1 \mathrm{MHz}$ chopping frequency with digital phase-sensitive detection software using a short time constant of $2 \mu \mathrm{s}$, preserving time resolution at the/targeted scale. The current and PMT signals are divided into blocks and transformed into the frequency domain with a discrete Fourier transform (DFT). The transfer function for each block is then calculated and averaged together to form $\langle H[k]\rangle$. After multiplying by the characteristic current trace, the resulting fluorescence trace $F[n]$ in the time domain is obtained with an inverse DFT. This technique assumes that the plasma source is a time-invariant linear system in which the linear transfer functions remain constant, and that the average estimated transfer function $\langle H[k]\rangle$ approaches the exact transfer function as the number of blocks increases. The method was demonstrated at a single point in a hollow cathode discharge with driven periodic oscillations $[67,35]$ and used to study IVDFs at three spatial locations and three maggnetic field settings in a $6 \mathrm{~kW}$ Hall thruster [29, 68].

2.3.4. Boxcar Method The Boxcar method for time-resolved LIF was proposed by N. A. MacDonald [69] of the Stanford Plasma Physics Laboratory (SPPL) and resembles the photon counting methodology. While the laser scans slowly, long traces of discharge current, PMT signal, and chopper signal are stored at high resolution and digitally post-processed.) PMT and current signals are partitioned into oscillation cycles $(\sim 40$ during a period of essentially constant laser wavelength) that are defined by locating current peaks, a technique used by Smith [72] for synchronizing time-resolved plasma probe data with discharge current oscillations. PMT cycles that occur while the laser is illuminating the discharge (fluorescence plus background signal) are averaged 
together, while those that occur while the laser is blocked by the mechanical chopper (just background) are averaged and subtracted from the total. Cycles in which the chopper transitions states partway through are discarded. After the time history of the fluorescence trace is obtained for each discrete laser wavelength, the time evolution of the IVDF is constructed by grouping the data from all wavelengths into common time bins. A baseline offset for each IVDF requires correction. The method has the advantage of obtaining full time and velocity space resolution in a single laser scan, reducing test time, with the time resolution limited by the data digitation rate, resolution, and storage capacity.

After applying the method to two numerical simulations of experiments with current oscillations at $60 \mathrm{~Hz}$ (tabletop xenon discharge tube) and $3 \mathrm{kHz}$ (target frequency range for a plasma thruster measurement), MacDonald concluded that while the Boxcar method worked well for perfectly coherent discharge current oscillations (constant frequency spectra in time), the sample-hold method (described below) held more promise for interrogating quasi-periodic systems where oscillations may not be exactly repeatable. In this situation, the Boxcar method would require an extra step of stretching or compressing the signals to a common cycle duration (period) for the time domain addition/subtraction process to work, adding noise.

2.3.5. Sample-Hold Method The sample-hold (also known as sample-and-hold) method for achieving time resolution with $\operatorname{LIF}$ was also developed at the SPPL $[69,56]$ and first demonstrated on a $60 \mathrm{~Hz}$ discharge [33]. It has since been applied to variety of EP systems, including cusped field thrusters [56] and Hall thrusters [30, 57, 60], enabling time-resolved IVDFs to be obtained in several operating conditions and spatial locations around the thruster channel and plume. Thís technique is applicable to both driven and naturally oscillating discharges, tolerating some amount of frequency drift in the system, and is described in much further detail elsewhere $[60,36]$. This method is used for the extensive measurements on the BHT-600 commercial Hall thruster presented in Section 3. Its primary adyantages lie in its scalable hardware implementation and similarity to the standard time-averaged LIF process that makes for easy reuse of existing methodologies, data analysis software, and experimental intuition.

As illustrated in Fig. 2, sample-hold processing is applied to the raw PMT signal, before the lock-in amplifier extracts the fluorescence excitation lineshape. Essentially, only signal that originates during a short time window (that is synchronized to the phase of the reference oscillation in the plasma source) is allowed to pass to the lockin, ensuring that the resulting LIF trace is representing only that single time interval. The method achieves high resolution in laser wavelength/velocity space and must be repeated for each time point to build up the evolution of the LIF lineshape.

Usually the plasma discharge current is used as the representative oscillating signal, although other sources like optical emission collected with a photodiode or a periodic current or voltage spike recorded by a physical probe in the discharge could also be used. The diseharge could even be pulsed assuming repeatable physics occur after each event. 


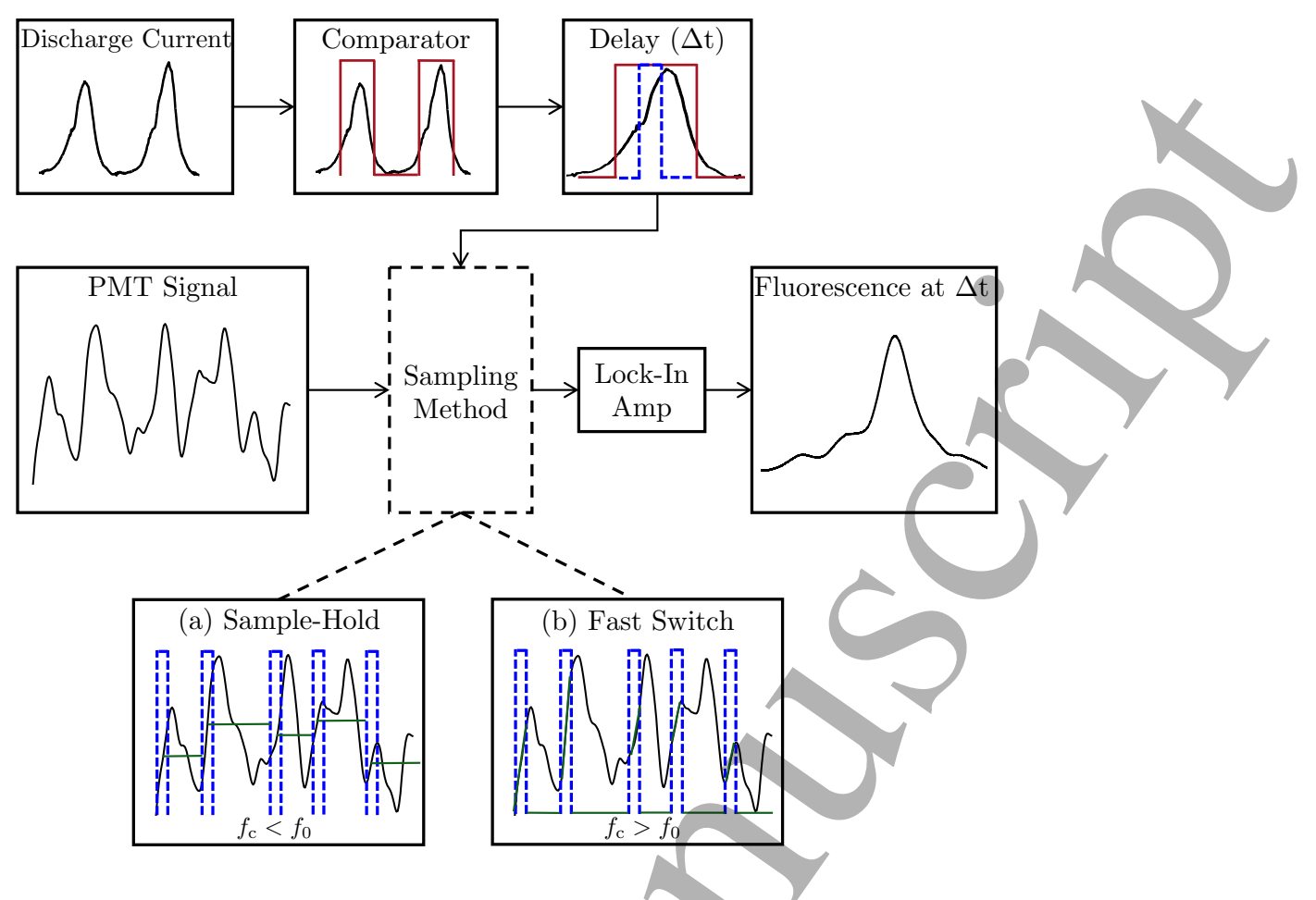

Figure 2. Block diagrams of the (a) sample-hold, and (b) fast switching timeresolution methods for laser-induced fluorescence spectroscopy. A comparator chip triggers the creation of a measurement gate of width $\tau$ and delay time $\Delta t$ from the point at which the discharge current crosses a defined threshold. The fluorescence signal from the PMT is sampled according to either method and fed into a lock-in amplifier that extracts the LIF lineshape at the chosen phase in the current cycle.

Regardless of the method employed, a trigger must be generated once per oscillation cycle that becomes the $t=0$, "zero phase" reference point. In the current version of the sample-hold systems installed at the Stanford Plasma Physics Laboratory (SPPL) and Air Force Research Laboratory (AFRL), a voltage comparator circuit provides the zero phase trigger (red in Fig. 2) when the discharge current signal passes through a certain threshold.

Once the zero phase trigger is generated, once per oscillation cycle, a digital delay generator produces a TTL pulse (blue in Fig. 2) of width $\tau$ that is delayed $\Delta t$ from the comparator trigger. The fluorescence signal collected inside each acquisition gate is averaged by a dedicated circuit and held until the following gate, at which point it is updated with the new value (green in Fig. 2a). This effectively rejects all other signal originating outside the desired phase in the current cycle, while maintaining a nonzero input signal level to the lock-in amplifier that proceeds with phase-sensitive homodyne detection. The acquisition gate is parked at the desired phase of the oscillation period and a full laser wavelength scan is performed, reconstructing the LIF transition lineshape at the specific time delay $\Delta t$. The time evolution of the LIF lineshape is then obtained by repeating laser scans at various delay times along the full current cycle. Since all time information is contained within the PMT signal during a single laser scan, 
multiple sample-hold branches, each with their own dedicated sample-hold circuit and lock-in amplifier, may be utilized to extract LIF lineshapes at several different delays $\Delta t$ simultaneously in parallel.

The optical chopping frequency $f_{\mathrm{c}}$ employed for this method is lower than the characteristic frequency $f_{0}$ of the plasma oscillation so that multiple sample-and-hold signal updates occur within one laser modulation. Typically $f_{0} / f_{\mathrm{c}} \approx 4-25$, providing the lock-in with sufficient variation in the fluorescence signal despite the averaging. A laser modulation frequency of $f_{\mathrm{c}}=1-2 \mathrm{kHz}$ is typically used for Hall thrusters with breathing mode frequencies in the $f_{0}=20-50 \mathrm{kHz}$ range. To date, the sample-hold method has only been demonstrated on systems with up to $50 \mathrm{kHz}$ oscillations; however, the measurement technique could be extended to higher frequencies (hundreds of $\mathrm{kHz}$ or higher) with the proper hardware.

2.3.6. Fast Switching Method The fast switching method, also developed by the SPPL $[70,60]$, operates much in the same way as the sample-hold technique. As illustrated in Fig. 2, the only difference arises in the way the PMT signal is sampled inside the measurement gate before entering the lock-in. In this method, the instantaneous PMT signal is allowed to pass unmodified to the lock-in during the measurement window, but is cut off to a null value by a fast semiconductor switch outside of the target phase range of the oscillation. The switch is triggered by the measurement gates produced by the pulse delay generator. The laser chopping frequency must now be much higher than the characteristic frequency of the discharge $\left(f_{\mathrm{c}} / f_{0} \approx 50-100\right)$ to provide the lockin with a clear frequency component at $f_{c}$. This procedure has the added benefit of operating in a higher frequency regime where the background spectral noise density is usually lower. The method has been applied to a $60 \mathrm{~Hz}$ evaluation study applying a laser chopping frequency of $f_{\mathrm{c}}=4.6 \mathrm{kHz}$. For use with Hall thrusters and other higher frequency systems, an acousto-optic device may be used for modulating the probe laser beam up to a few $\mathrm{MHz}$, as demonstrated previously [62, 35].

\section{Application: Time-Resolved Velocimetry of a BHT-600 Hall Thruster}

In this section, the sample-hold method of Section 2.3.5 is applied to a time-resolved LIF velocimetry diagnostic of a commercial BHT-600 Hall thruster. The thruster operates in a quasi-periodic mode that supports a strong breathing oscillation [16, 17] measurable in the channel of the device, By leveraging hardware parallelization of the sample-hold measurement, we are able to significantly increase the data acquisition rate over previous instantiations of the system $[30,56,33]$ and capture the ionization and propellant acceleration dynamics of the breathing mode with high spatial and temporal resolution. 


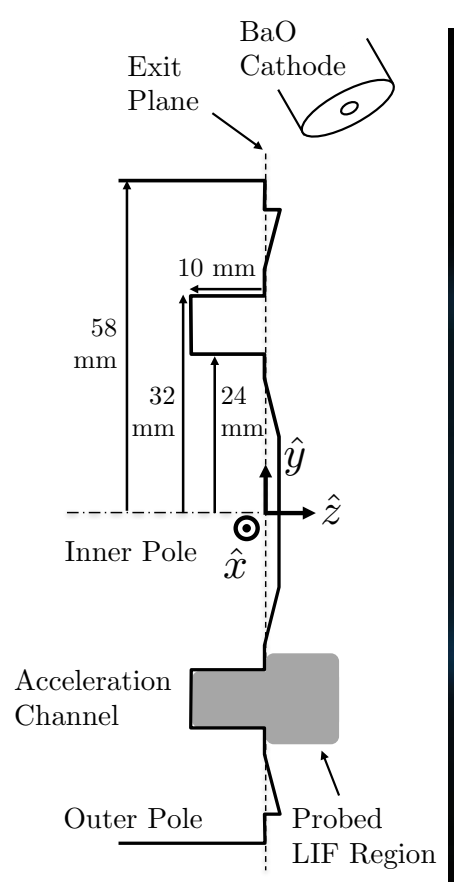

(a)

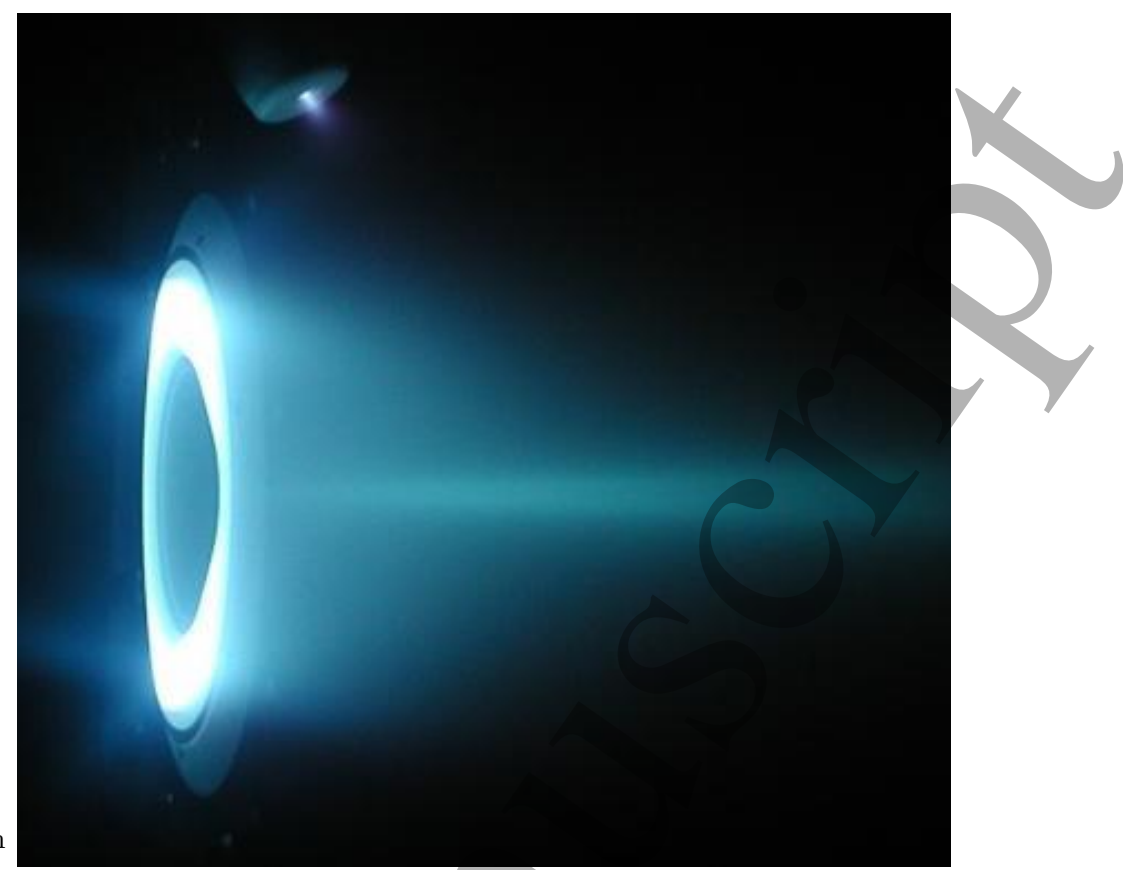

(b)

Figure 3. (a) Diagram of BHT-600 Hall thruster, including thruster dimensions and the coordinate system used in this work. (b) Side view of BHT-600 Hall thruster operating on xenon. The external cathode is visible above the thruster.

\subsection{BHT-600 Hall Thruster}

The BHT-600 is a high-efficiency mature propulsion system that nominally produces $39 \mathrm{mN}$ of thrust with a specific impulse of $1500 \mathrm{~s}$ at $600 \mathrm{~W}$ of power [73]. The thruster can operate between 200-800 W with xenon, krypton, or iodine propellants, and uses an externally mounted BHC-1500 barium oxide hollow cathode for plume neutralization. The thruster mass is $2.6 \mathrm{~kg}$, while the cathode mass is $0.2 \mathrm{~kg}$. The thruster features a short ion acceleration zone located midway along the discharge channel of outer radius $32 \mathrm{~mm}$, inner radius $24 \mathrm{~mm}$, and depth $10 \mathrm{~mm}$. The magnetic field is produced by four outer magnetic coils and one inner coil, which is operated independently to optimize the field topology. Figure 3 shows a side view of the BHT-600 operating on xenon, along with the dimensions and coordinate system used for this study. This thruster has been studied extensively using both electrostatic probes and various optical diagnostics [74, 75, 76].

The thruster is operated at $300 \mathrm{~V}$ anode potential with $2.21 \mathrm{mg} / \mathrm{s}(22.5 \mathrm{sccm})$ of xenon propellant flow. In this condition the BHT-600 draws 2.05-2.15 A of discharge current, with some drift observed in day-to-day operation. The cathode consumes $147 \mu \mathrm{g} / \mathrm{s}(1.5 \mathrm{sccm})$ of xenon propellant. Both magnet coils are operated with $1.75 \mathrm{~A}$. With these settings, the BHT-600 experiences breathing mode oscillations of the discharge current in the $43-50 \mathrm{kHz}$ range. Anode current traces reveal a daily drift in the oscillation frequency, with the lowest observed to be $44 \mathrm{kHz} \pm 0.6 \mathrm{kHz}$ and the 
highest observed to be $49 \mathrm{kHz} \pm 1.1 \mathrm{kHz}$. Here, the uncertainty is given as the standard deviation of a Gaussian profile fit to the fundamental frequency obtained from an FFT. One representative discharge current trace and its spectral content is shown in Fig. 4, as well as the measured anode voltage and typical optical emission signal recorded by a photomultiplier tube (PMT). At the time of measurement, the BHT-600 is oscillating at $48 \mathrm{kHz}$ with a mean cycle period of $20.8 \mu \mathrm{s}$. The discharge voltage and current traces are offset by $180^{\circ}$ in phase. The negative PMT signal increases in phase with the current, indicating greater background excited ion production and emission near the monitored wavelength of $542 \mathrm{~nm}$ when the discharge current is high. All three signals show a strong component at the breathing mode frequency of $48 \mathrm{kHz}$, in addition to higher harmonics.

(a)

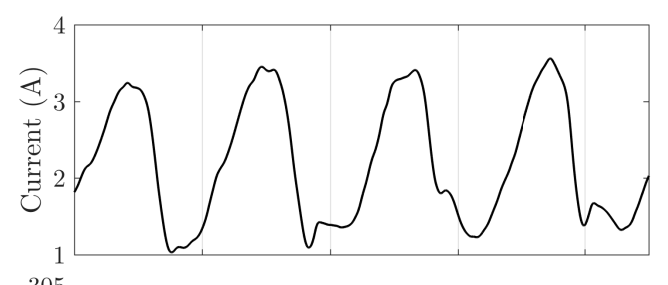

(b)

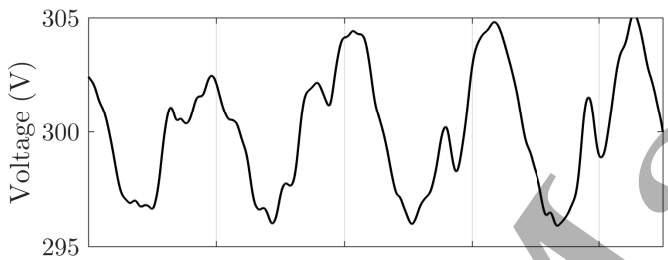

(c)

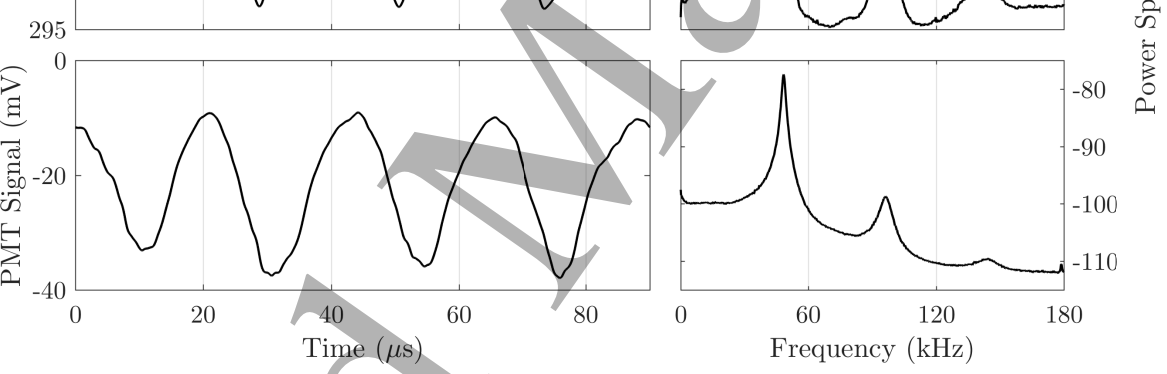

Figure 4. Left column: (a) discharge current, (b) discharge voltage, and (c) plasma emission signals captured from the BHT-600. Right column: spectral content of each signal showing the dominant breathing mode at $48 \mathrm{kHz}$. Note that the photomultiplier tube (PMT) signal is negative, indicating increasing plasma emission in the downward direction.

\subsection{BHT-600 Experimental Setup}

LIF measurements for this study are performed in Chamber 6 at the Air Force Research Laboratory (AFRL) at Edwards AFB, CA. This facility, described elsewhere [69], is capable of achieving a base pressure of around $10^{-6}$ Torr and a background pressure during thruster operation of about $4 \times 10^{-5}$ Torr (uncorrected for xenon). The $1.8 \mathrm{~m}$ diameter, $3 \mathrm{~m}$ long chamber is stainless steel, non-magnetic, and provides a xenon pumping speed of $32,000 \mathrm{~L} / \mathrm{s}$ using four single stage cryogenic panels $(25 \mathrm{~K})$ and one two-stage cryogenic pump $(12 \mathrm{~K})$. Three motorized translation stages mounted in 
perpendicular directions provide fine control over the thruster position to sub-millimeter precision.

The laser-induced fluorescence optical setup at AFRL $[77,54]$ follows the typical layout described in Fig. 1 and Section 2.2. Further detail is shown in Fig. 5. Xenon ion velocity measurements are performed by probing the $5 d[4]_{7 / 2}-6 p[3]_{5 / 2}^{0}$ Xe II transition ( $834.953 \mathrm{~nm}$ vacuum) with $\sim 23 \mathrm{~mW}$ of diode laser power. The probe beam, generated by a TA-7600 tapered amplifier seeded by a New Focus TLB-6817 tunable diode laser, enters the chamber through a window directly in front of the thruster for axial velocity measurements ( $-\hat{z}$ in Fig. 3 ), or is diverted through a second window (not shown) and turned $90^{\circ}$ with a mirror to run parallel to the exit plane for radial measurements $(+\hat{x}$ direction). The resulting fluorescence from the $6 s[2]_{3 / 2}-6 p[3]_{5 / 2}^{\mathrm{o}}$ transition at $542.1 \mathrm{~nm}$ (vacuum) is collected along a line $\sim 60^{\circ}$ from the main thruster axis, filtered through a $125 \mathrm{~mm}$ focal length monochromator with a bandwidth of a few nanometers, and transformed into an electrical signal with a Hamamatsu R928 PMT (15 k $\Omega$ shunt) for determining the Doppler shift of the fluorescence excitation spectrum. Two $100 \mathrm{~mm}$ diameter, $200 \mathrm{~mm}$ focal length lenses are used in this system with 1:1 magnification, and the intersection of the monochromator entrance slit geometry of $\sim 0.7 \mathrm{~mm} \times 1.5 \mathrm{~mm}$ with the sub-millimeter focused probe beam defines the $\sim 1 \mathrm{~mm}^{3}$ spatial resolution of the system.

Beam pick-offs send portions of the laser into a Fabry-Pérot (F-P) etalon for a relative frequency reference, and an optogalvanic xenon reference cell (OGC) for an absolute reference. The interferometer provides fixed frequency markers every FSR $=0.3 \mathrm{GHz}$. The $6 p^{\prime}[3 / 2]_{1}-8 s^{\prime}[3 / 2]_{1}$ Xe I transition ( $834.974 \mathrm{~nm}$ vacuum) in the optogalvanic cell, spaced $\sim 10 \mathrm{GHz}$ from the stationary target ion transition in the direction of the ion Doppler shift, is used for the zero velocity reference. This choice of reference line, instead of the more commonly used Xe I $6 s^{\prime}[1 / 2]_{1}^{0}-6 p^{\prime}[3 / 2]_{2}$ transition at $834.91 \mathrm{~nm}$ (vacuum) spaced $\sim 18 \mathrm{GHz}$ from the target line in the opposite direction, significantly reduces the time required to perform each laser scan.

The sample-hold method is used for obtaining time resolution in the measured ion velocity distribution function lineshapes. As shown in Fig. 5, the PMT voltage signal may be split $N$ ways into separate sample-hold circuit plus lock-in amplifier channels for simultaneous data collection. The data reported here were obtained using $N=6$ channels, enabling 24 total LIF lineshapes to be collected in four successive laser scans across the Doppler-shifted transition. One measurement is reserved for the timeaveraged LIF lineshape at each spatial location (bypassing the sample-hold circuit), leaving 23 points for time-resolved LIF data that are taken in $1 \mu \mathrm{s}$ increments with $1 \mu \mathrm{s}$ gate widths.

Time-resolved LIF data is obtained at multiple spatial locations in the $-\hat{y}$ portion of the BHT-600 channel, defined according to the coordinate system shown in Fig. 3. Note that the cathode is located above the channel in the $+\hat{y}$ direction, and the collection lens is located on the $-\hat{x}$ side of the thruster. The axially-oriented exciting laser is launched in the $\rightarrow \hat{z}$ direction, and the resulting Doppler shifts are plotted such that ions with a 


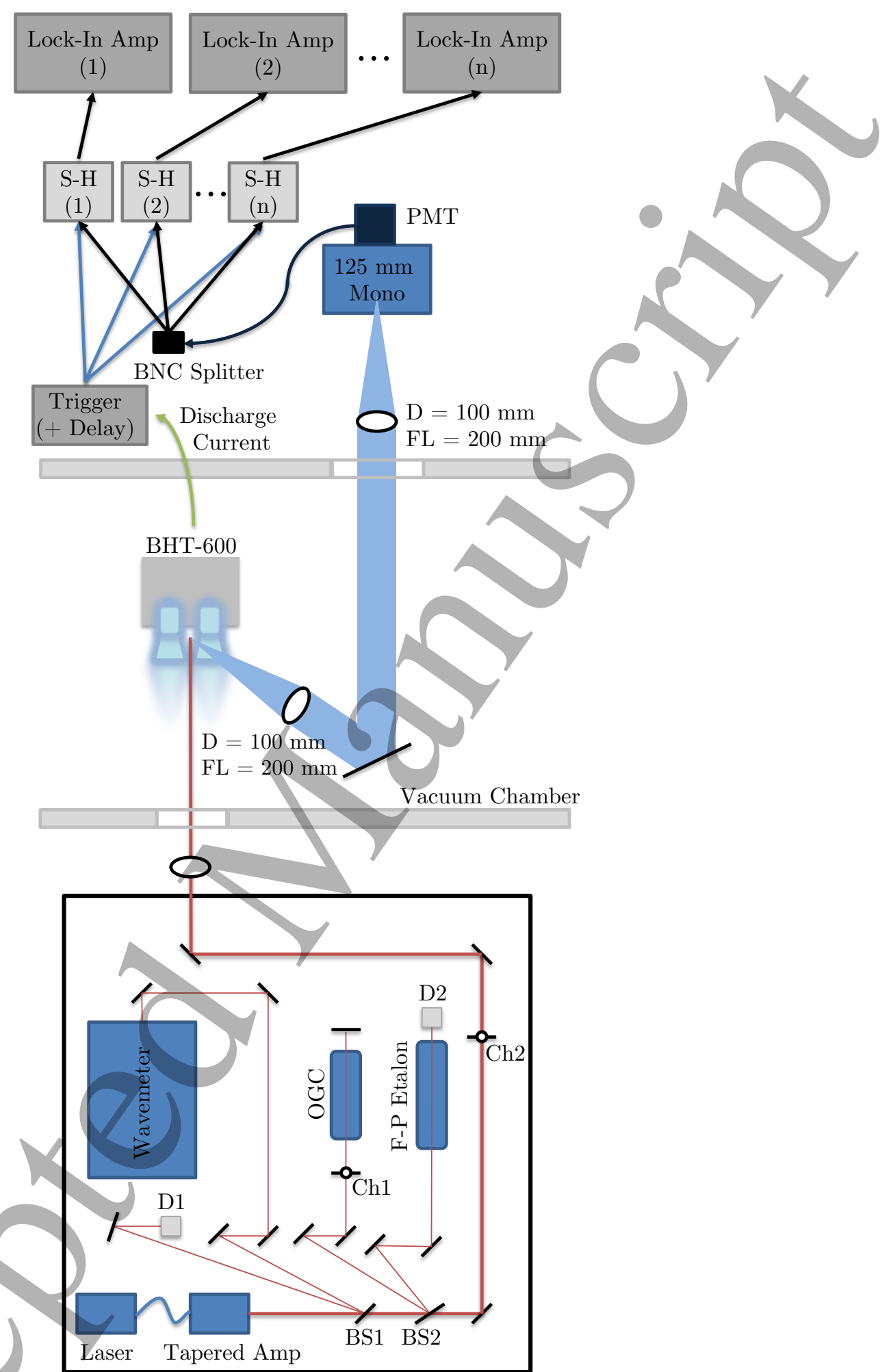

Figure 5. Configuration of the AFRL Chamber 6 time-resolved LIF experimental apparatus. The collection lenses are $100 \mathrm{~mm}$ in diameter with a focal length of $200 \mathrm{~mm}$, and the monochromator focal length is $125 \mathrm{~mm}$. Legend: BS $=10-90$ Beam Splitter, $\mathrm{CH}=$ Chopper, $\mathrm{D}=$ Photodiode, $\mathrm{F}-\mathrm{P}=$ Fabry-Pérot, $\mathrm{MONO}=$ Monochromator, OGC $=$ Optogalvanic Cell, PMT $=$ Photomultiplier Tube. 
positive velocity are traveling in the $+\hat{z}$ direction.

\subsection{Results and Discussion}

This section examines the time-averaged and dynamical behavior of the axial ion velocities in the $10 \mathrm{~mm}$ long BHT-600 channel. Ions are accelerated over a sharp potential gradient located in the downstream half of the channel, reaching close to their maximum velocity within the first few millimeters into the plume. This contrasts with the primarily external ion acceleration observed in other thrusters with different channel aspect ratios. One such device, the Stanford Z-70 Hall thruster [30,60] that closely resembles those of the SPT family, will be used here to make instructive comparisons with the present data.

3.3.1. Time-Averaged Figure 6 displays the time-averaged axial ion LIF data obtained along the channel centerline $(y=-28 \mathrm{~mm})$, both in the channel and/out into the plume. The dotted lines depict the time-averaged traces without sample-hold processing, while the solid lines show the integral average of the time-resolved traces (described in the following section). This offers a validation of the sample-hold method that it is indeed recovering accurate dynamic information over the whole oscillation period. It is clear from the time-averaged data that the greatest ion/acceleration occurs between about $z=-4 \mathrm{~mm}$ and $z=0$, with some additional gain in velocity achieved out into the plume. Here, the ion velocity distribution functions (IVDFs) experience substantial broadening. This effect is likely due to the breathing mode dynamics of a moving ionization and acceleration front traversing this area, as described in the following section. As shown in Ref. [30], a narrow IVDF moving in time can make the timeaveraged trace appear broadened. Not much additional acceleration is achieved in the plume past $z=\sim 6 \mathrm{~mm}$. The highest LIF intensity, proportional to the metastable ion lower excited state population density, is observed near $z=-6 \mathrm{~mm}$, indicating that this region is likely the primary zone of ionization in the BHT-600. There is no evidence of low velocity ion populations in the first centimeter of the plume in the time-averaged data.

The time-averaged ion velocities measured here can be used to validate the predatorprey prediction of the BHT-600 breathing mode frequency using Eqn. 1. Figure 6 shows ions accelerating to a velocity of roughly $16 \mathrm{~km} / \mathrm{s}$ across an $8 \mathrm{~mm}$ section of the discharge channel. Assuming a time-averaged neutral velocity of around $350 \mathrm{~m} / \mathrm{s}$ (suggested by neutral xenon LIF measurements of the Z-70 Hall thruster and other similar discharges, see Fig. 6 of Ref. [30] for example), a simple estimate of the breathing mode frequency yields $f_{\mathrm{b}}=47 \mathrm{kHz}$, within the range of observed values between $44-49 \mathrm{kHz}$.

3.3.2. Time-Resolved Moving to the time-resolved data, Fig. 7 shows the variation in most probable ion velocity (a) and peak lineshape intensity (b) over the period of a typical discharge current oscillation (c) along the thruster acceleration channel 


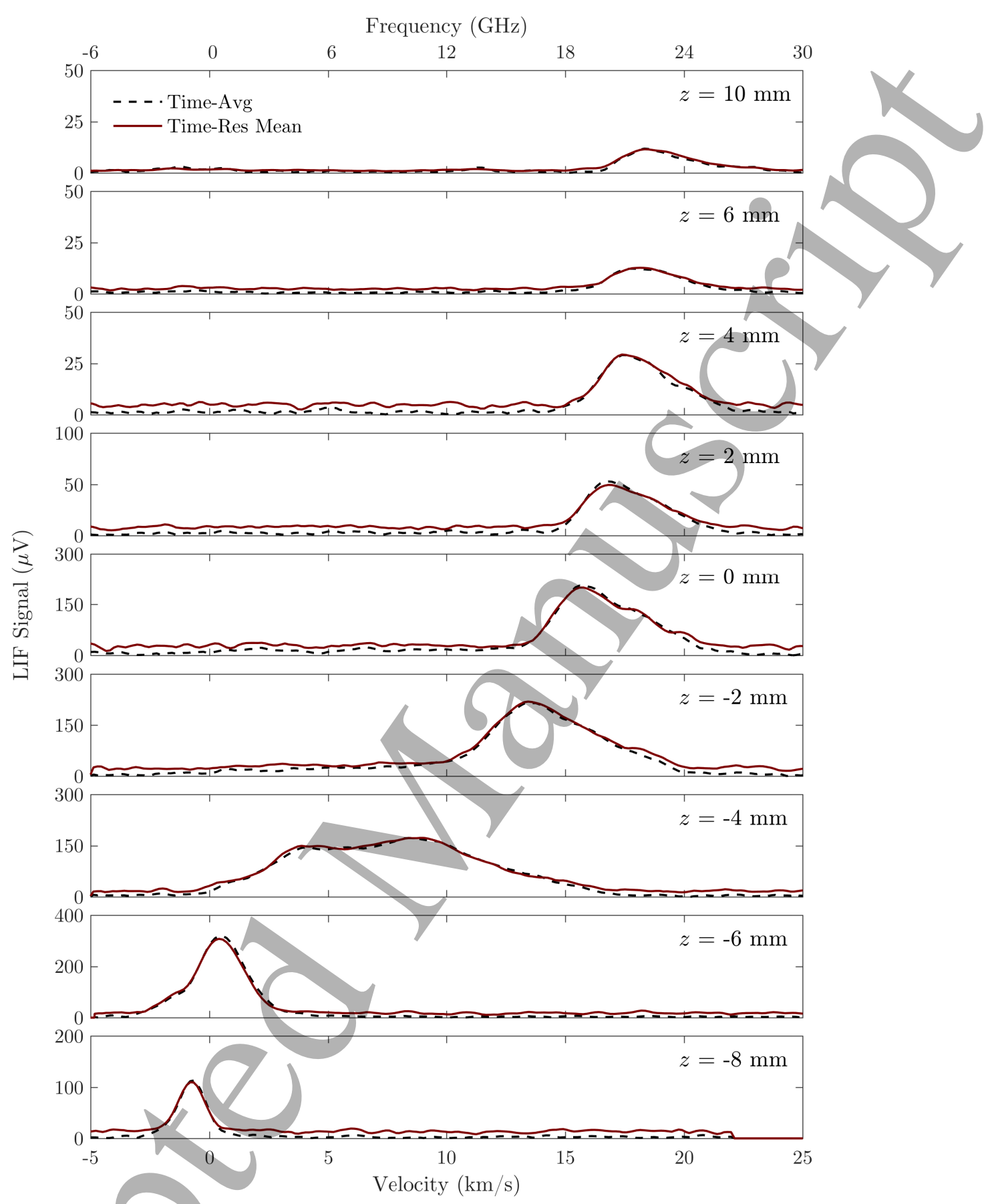

Figure 6. Time-averaged LIF traces of the axial ion velocity along the BHT-600 channel centerline $(y=-28 \mathrm{~mm})$. The anode is at $z=-10 \mathrm{~mm}$ and the exit plane is at $z=0$. The integral average of the time-resolved traces (solid lines) matches the time-averaged traces (dotted lines) taken without sample-hold processing. 


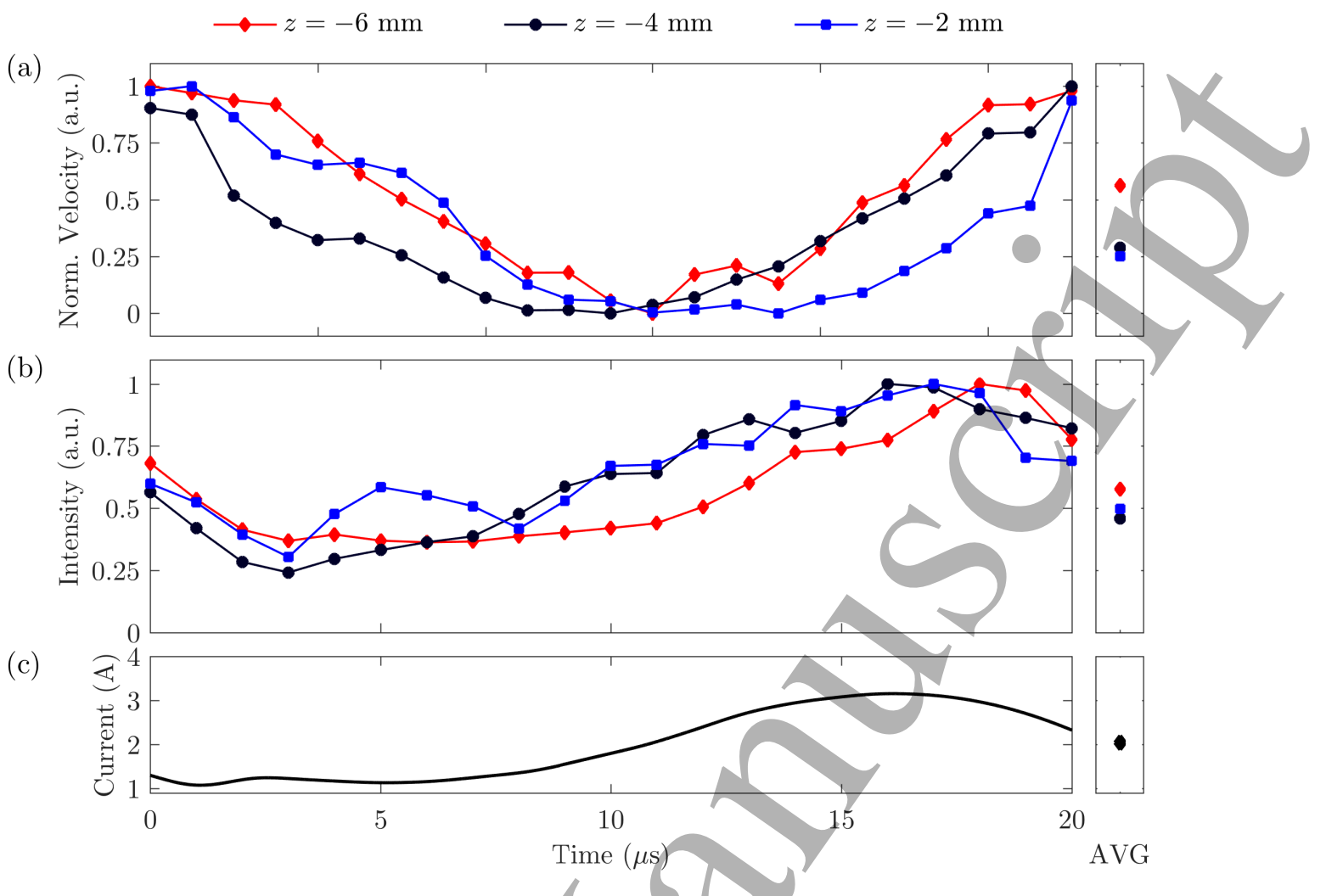

Figure 7. Trends along the BHT-600 acceleration channel centerline for (a) most probable axial ion velocity normalized by the total variation: $\left(V-V_{\min }\right) /\left(V_{\max }-V_{\min }\right)$, and (b) peak fluorescence excitation lineshape intensity normalized by the maximum value over the (c) discharge current cycle. The boxes at right show the equivalent results from a time-averaged measurement.

centerline $(y=-28 \mathrm{~mm})$. The boxes at right show equivalent results obtained from timeaveraged LIF velocimetry. The most probbable ion velocities are shown here normalized by the total variation over the cycle for each location: $\left(V-V_{\min }\right) /\left(V_{\max }-V_{\min }\right)$. This highlights the similar profile of the velocity modulations over time, despite differing overall magnitudes as the ions accelerate downstream. Throughout the channel, the velocities reach their maxima about one quarter oscillation period after the discharge current peak $\left(90^{\circ}\right.$ phase lag). The velocities then fall, reaching their minima as the discharge current begins its next ramp up.

The variations in the peak intensity of the measured fluorescence excitation lineshapes also follow the same overall trend throughout the acceleration channel. The intensity profiles closely track the current oscillation, and at some spatial locations the small inflections seen in the first $8 \mu$ s of the current trace can be observed in both the intensity and velocity plots. The relative fluorescence intensity at a given phase in the current cycle is related to the instantaneous metastable Xe II probed state density $\left(5 d[4]_{7 / 2}\right)$, which is linked to the ground state xenon ion density through complex collisional-radiative processes [31, 32].

It is informative to compare these results with simulations and similar 
measurements made on Hall thrusters with different channel geometries and operating conditions. Upon comparison with Ref. [30], one will notice similarities in the ion velocity and intensity modulations between the BHT-600 and the Stanford Z-70 Hall thrusters: the main ion population achieves maximum velocity in the current trough and the fluorescence intensity is in phase with the current. This pattern is in agreement with other probe-based breathing mode studies [23] and numerical models [78, 17, 16, 79, 80]. For example, Bareilles et al. [78] used a two-dimensional (radial-axial) particle-in-cell (PIC) code to study strong ionization oscillations in a Hall thruster similar to the SPT-100. Figures 6 and 7 of that work, along with the associated discussion, identify the progression of events that are generally understood to give rise to breathing mode oscillations. Other pertinent results include Figs. 2-4 of Barral and Ahedo [17], Figs. 810 of Boeuf and Garrigues [16], Figs. 3 and 5 of Hara et al. [79], and Figs. 6-8 of Yang et al. [80].

The discharge current increases as the ionization front moves upstream, consuming neutral propellant and increasing the population of free electrons. The fluorescence intensity consequently increases as the ion population grows, and the ionization rate reaches its maximum in the peak magnetic field region. (For the simulated thruster, this is near the exit plane and the ion acceleration front extends outside the thruster; in the BHT-600 this is essentially confined within the $10 \mathrm{~mm}$ channel.) At the discharge current peak, the ionization rate is maximized and the ionization zone spreads over a larger volume of the discharge channel, strongly depleting the neutral population. The newly generated ions accelerate out of the channel according to the local potential field, which is also changing with time. Ions obtain their maximum velocity after the point of peak ionization as the electric field peaks, and the ion density (and measured intensity) necessarily falls as the ions accelerate. The ionization front moves back downstream during the discharge current trough, the neutral population builds, and the cycle repeats as the discharge current ramps up again.

Further insight is gained from plotting the evolution of the IVDFs over the full channel in Fig. 8. LIF scans collected over a gate width of $1 \mu$ s are arranged vertically in the contour plots, maintaining their relative signal intensity, and each panel is normalized by the overall maximum value. The data taken over one cycle are repeated to evoke the periodic nature of the modulations. The anode is located at $z=-10 \mathrm{~mm}$ (bottom of the figure), the exit plane is at $z=0$ (top), the inner channel wall is at $y=-24 \mathrm{~mm}$ (left), and the outer channel wall is at $y=-32 \mathrm{~mm}$ (right). The contour plots highlight the spatial and temporal extent of ion acceleration in the BHT600 channel. Only minimal radial variations are observed in the time-resolved velocity profiles, indicating a primarily $1 \mathrm{D}$ axial ion acceleration in the channel. At $z=-8 \mathrm{~mm}$ near the anode, the magnitude of the velocity modulation is small and some ions attain a slightly negative velocity. This behavior can be attributed to a gradient-driven field reversal, often seen in hybrid Hall thruster simulations [81, 82]. Moving downstream to $z=-6 \mathrm{~mm}$, the velocity distributions widen slightly and noticeable temporal variations become apparent. 


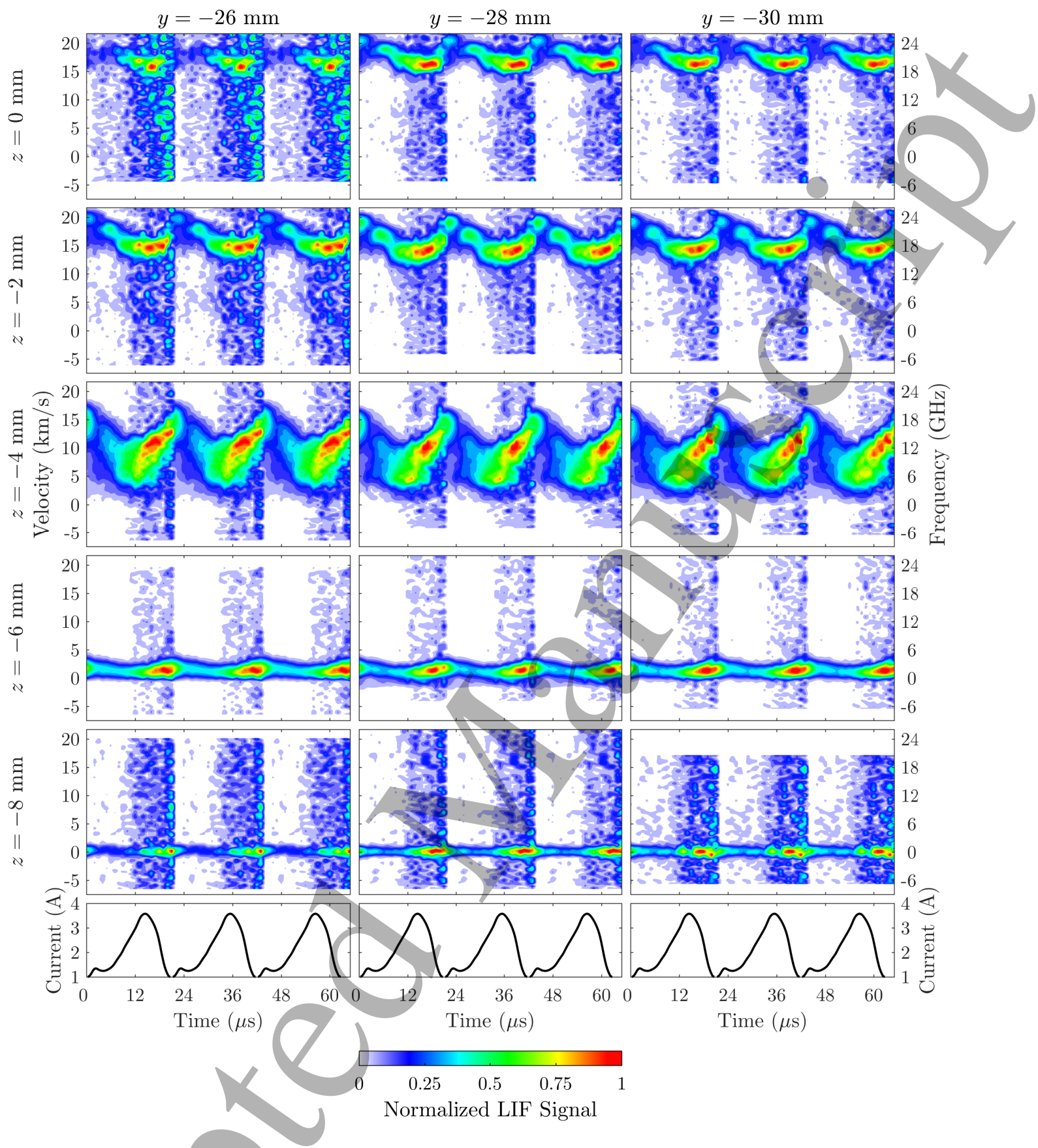

Figure 8. Time-varying axial ion velocity distributions for a typical breathing mode current cycle (repeated three times for effect) at several locations in the thruster channel. The anode is at $z=-10 \mathrm{~mm}$ and the exit plane is at $z=0$. The current trace orients the time axis in the bottom panels. The contour plots are normalized to the peak LIF signal intensity in each frame. The maximum signal intensities moving from $z=-8$ to $z=0$ along $y=-28 \mathrm{~mm}$ are $195,550,375,435$, and $435 \mu \mathrm{V}$, respectively. 
The majority of channel acceleration occurs near $z=-4 \mathrm{~mm}$, where the velocity distributions broaden significantly and show large temporal variations over the course of the current cycle. This suggests that both the spatial extent of propellant ionization and local potential significantly fluctuate in this region, with the resulting most probable ion velocities ranging between $6-14 \mathrm{~km} / \mathrm{s}$. Closer to the channel exit at $z=-2 \mathrm{~mm}$ and $z=0$, the IVDFs narrow again and the magnitude of the velocity fluctuation is less pronounced, indicating that by this location the majority of ions have been/accelerated evenly. Moving out into the plume, the temporal velocity modulations continue to diminish in strength and not much additional ion acceleration occurs.

Figure 9 illustrates the progression of a moving acceleration and ionization front in the channel over the breathing mode cycle, with the time-averaged data appearing at the bottom for comparison. Here, the most probable velocity (left column), calculated axial electric field (middle column), and peak LIF intensity (right column, surrogate for metastable ion density) are plotted over the channel and out into the near field at several different snapshots in time. The corresponding discharge current trace may be found in Figs. 7 and 8. LIF measurement points are indicated by symbols $(\boldsymbol{\Lambda})$.

Using the most probable velocities, $v_{z}$, obtained from the LIF peak locations, one can estimate the time-resolved axial electric field $E_{z}(t)$ in the BHT-600 channel with the following equation $[28,10]$ :

$$
E_{z}(t)=\frac{m_{\mathrm{i}}}{e}\left(\frac{\partial v_{z}}{\partial t}+v_{z} \frac{\partial v_{z}}{\partial z}\right)
$$

where $m_{\mathrm{i}}$ is the xenon mass, $e$ is the fundamental charge. This simplified equation may be derived from the ion momentum equation and assumes a steady-state, 1D, collisionless plasma in the axial direction, with either cold ions or a uniform ion density in $\hat{z}$ to cancel the pressure tensor (a major assumption). Thus, this should be taken as a rough approximation only. Further discussion on calculating electric fields from LIF data may be found in Refs. [28, 83, 84]. Small negative values in the electric field near the anode introduced through numerical noise and uncertainty in the velocity measurement have been forced to zero.

The measured or calculated quantities are interpolated throughout the remainder of the domain using an inverse-distance method [33], where $\phi_{\mathrm{s}}$ are the source points known from LIF and $\phi_{\mathrm{d}}$ is the interpolated value at the destination point:

$$
\begin{gathered}
\phi_{\mathrm{d}}=\frac{\sum w_{\mathrm{s}} \phi_{\mathrm{s}}}{\sum w_{\mathrm{s}}} \\
w_{\mathrm{s}}=D^{-E} .
\end{gathered}
$$

The weighting function $w_{\mathrm{s}}$ is determined by the distance $D$ between the source and destination points and a weighting factor $E$, chosen here to be 3.5 [33]. This value gives more weight to immediately adjacent source points and yields good smoothing. The white boxes in the upper and lower portion of each panel represent the channel walls.

A clear picture of the breathing mode emerges from this viewpoint, in agreement with simulation-based studies from the literature $[78,17,16,79,80]$. (A particularly 


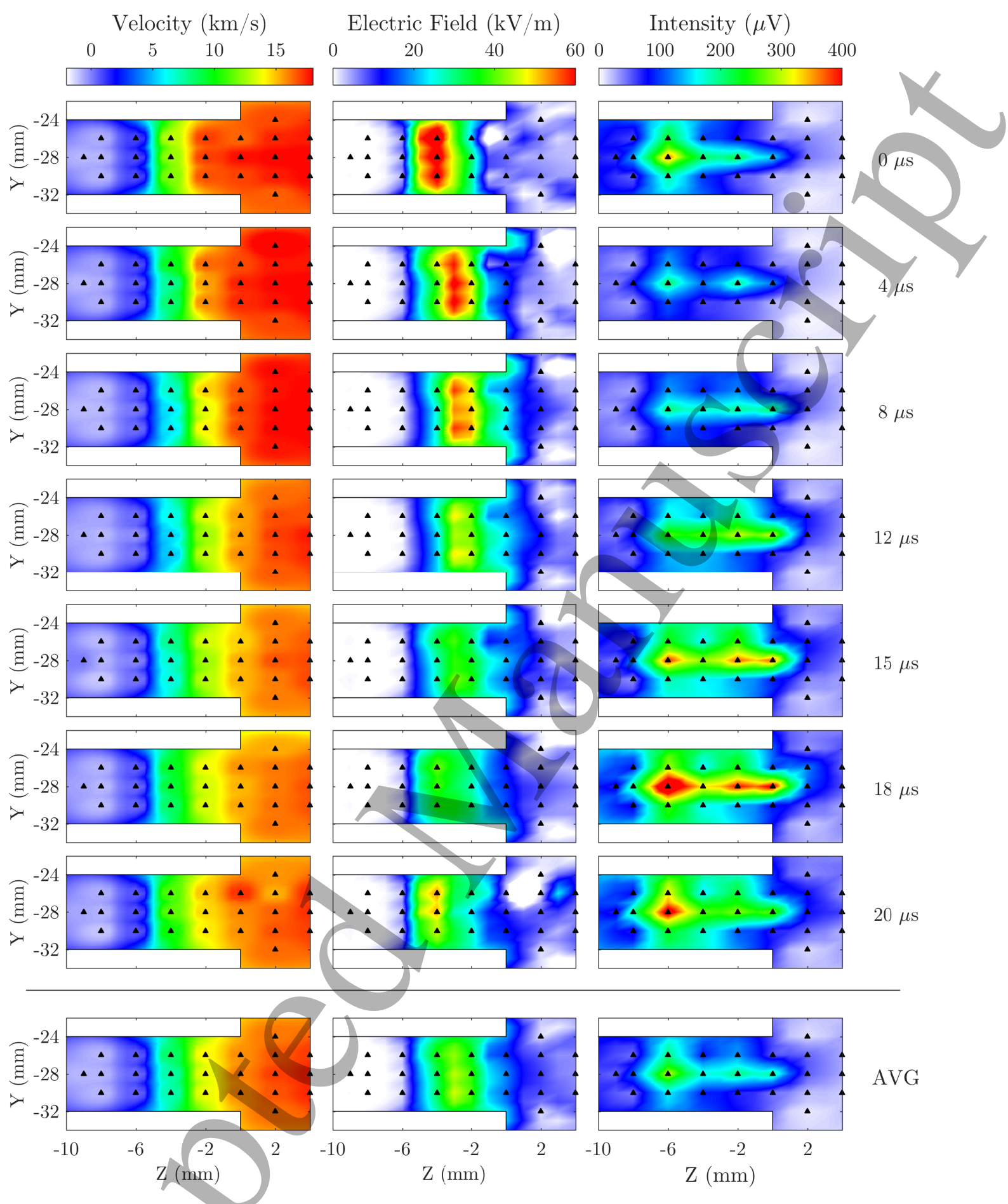

Figure 9. Time-resolved most probable axial ion velocity, electric field, and peak LIF intensity throughout the channel and near-field plume of the BHT-600 presented at several snapshots in time. The corresponding (intensity-weighted) time-averaged data appears at the bottom for comparison. The channel walls are represented as white boxes. 
effective comparison is again made against Fig. 7 of Bareilles et al. [78], noting that the discharge current peak at $99 \mu \mathrm{s}$ in that study corresponds to $\sim 16 \mu$ s here.) As the discharge current ramps up between $\sim 8-14 \mu \mathrm{s}$, the accelerating potential drop (electric field peak) is located furthest downstream. The channel has been refilling with neutrals before the start of the next cycle, plume velocities are lowest (some accelerated ions from the previous cycle are still apparent at $t=8 \mu \mathrm{s}$ ), and the metastable ion density (LIF intensity) begins to build out of the minimum in the trough. Then, more ions are produced and accelerated as the ionization front and potential drop progress upstream during the $\sim 14-20 \mu \mathrm{s}$ interval of peak discharge current. Ion production seems to be concentrated in the center of the channel, peaking at $18 \mu \mathrm{s}$ at $z=-6 \mathrm{~mm}$, upstream of the region traversed by the oscillating electric field and accelerating potential. As the current falls past $20 \mu \mathrm{s}$ to $\sim 2 \mu \mathrm{s}$ after the trigger, the electric field peaks and the acceleration zone reaches its maximum extent upstream. Here, ions are strongly accelerated over a short distance between $z=-5$ and $z=-3 \mathrm{~mm}$, and plume velocities approach their maximum. Ion density begins to fall as ions accelerate away from the channel and the ionization rate slows with the depletion of the neutral propellant. Finally, the acceleration zone moves downstream again while the current is minimum and neutrals refill the channel between $t=2-8 \mu \mathrm{s}$. Ions remaining in the near-field plume have achieved maximum velocity due to the recent peak in electric field, and the LIF intensity is lowest.

This pattern of time variation in the thruster electric field has also been reproduced by the two-dimensional particle-in cell (PIC) simulations of Yang et al., as illustrated in Fig. 8 of Ref. [80] and described in the associated discussion. The authors observe the maximum electric field during the current trough and the minimum electric field near the time of maximum plasma density.

Finally, the time-averaged data at the bottom of Fig. 9 shows how the narrower time-dependent features have been smeared over a larger area with reduced peak magnitude in the average. Recall that this data is an intensity-weighted average of the time-resolved features, so the data obtained in the high current interval is more heavily weighted.

\section{Conclusion}

In recent years, several techniques have been developed worldwide to perform timeresolved LIF measurements in oscillating plasmas. One of the main applications of these novel architectures is the study of plasma fluctuations in Hall thrusters, achieving highfidelity, nonintrusive measurements of the time evolution of the ion velocity distribution function (IVDF). This paper compares and contrasts these techniques in detail and applies one of the methods, the sample-hold approach, to the study of a commercial BHT-600 Hall thruster undergoing unforced quasi-periodic breathing mode oscillations.

Time-resolved continuous wave LIF measurements of metastable xenon ion velocities reveal subtle details about the physical mechanisms governing ionization and 
ion acceleration in this device. A parallelized sample-hold scheme with multiple data acquisition channels enables collection of a large dataset of time-resolved information throughout the channel at a single operating condition. Measurements are synchronized with the $44-49 \mathrm{kHz}$ breathing mode oscillation characteristic of such thrusters.

The evolution of the measured ion velocity distributions demonstrates the primarily one dimensional nature of the ionization and subsequent ion acceleration processes in the $10 \mathrm{~mm}$ deep annular channel. The dataset shows exactly where strong ionization occurs and enables characterization of an evolving electric field that is periodic with the breathing mode current oscillation. Very broad time-averaged TVDFs around $z=-4 \mathrm{~mm}$ arise from much narrower time-resolved IVDFs with a very strong intensity and velocity modulation in the zone of primary ion acceleration. Several locations in the channel exhibit an intensity modulation that lags the discharge current by a few microseconds, while the ion velocities lag the discharge current by a quarter cycle. This behavior is explained by the time-resolved velocity data and a careful consideration of the spatiotemporal history of the thruster acceleration zone throughout the breathing mode cycle.

\section{Acknowledgments}

This work is sponsored by the U.S. Air Force Office of Scientific Research with Dr. M. Birkan as program manager. C.Y. acknowledges support from the DOE NNSA Stewardship Science Graduate Fellowship under contract DE-NA0002135 and the Stanford Graduate Fellowship.

\section{References}

[1] Jahn R G 1968 Physics of electrie propulsion (McGraw-Hill, Inc., New York)

[2] Ronald W Humble Gary N Henry W J L 1995 Space Propulsion Analysis and Design (McGraw-Hill Companies, Inc.)

[3] Choueiri E Y 2004 Journal of Propulsion and Power 20 193-203

[4] Mazouffre S 2016 Plasma Sources Science and Technology 25033002

[5] Cappelli M A 2009 Physics Today 6276

[6] Kim V 1998 Journal of Propulsion and Power 14 736-743

[7] Koppel C R and Estublier D 2005 The smart-1 hall effect thruster around the moon: In flight experience Proceedings of the 29th International Electric Propulsion Conference IEPC-2005-245

[8] Delgado J J, Baldwin J A and Corey R L 2015 Space systems loral electric propulsion subsystem: 10 years of on-orbit operation Proceedings of the 34th International Electric Propulsion Conference IEPC-2015-04

[9] Casaregola C 2015 Electric propulsion for station keeping and electric orbit raising on eutelsat platforms Proceedings of the 34th International Electric Propulsion Conference IEPC-2015-97

[10] Vaudolon J, Khiar B and Mazouffre S 2014 Plasma Sources Science and Technology 23022002 URL http://stacks . iop.org/0963-0252/23/i=2/a=022002

[11] Hargus, Jr W A and Cappelli M A 2001 Applied Physics B 72 961-969

[12] Mazouffre S 2013 Plasma Sources Science and Technology 22013001

[13] Meezan N B, Hargus, Jr W A and Cappelli M A 2001 Physical Review E 63(2) 026410

[14] Janes G and Lowder R 1966 Physics of Fluids 9 1115-1123 
[15] Zhurin V, Kahn J, Kaufman H, Kozubsky K and Day M 1993 Dynamic characteristics of closed drift thrusters Proceedings of the 23rd International Electric Propulsion Conference IEPC-1993095 (Seattle, WA)

[16] Boeuf J P and Garrigues L 1998 Journal of Applied Physics 843541

[17] Barral S and Ahedo E 2009 Physical Review E 79046401

[18] Fife J M, Martinez-Sanchez M and Szabo J 1997 A numerical study of low-frequency discharge oscillations in Hall thrusters Proceedings of the 33rd AIAA/ASME/SAE/ASEE Joint Propulsion Conference \& Exhibit (Seattle, WA)

[19] Barral S and Peradzynski Z 2009 A new breath for the breathing mode Proceedings of the 31 st International Electric Propulsion Conference IEPC-2009-070 (Ann Arbor, MI)

[20] Hara K, Sekerak M J, Boyd I D and Gallimore A D 2014 Physics of Plasmas 21122103

[21] Lobbia R B 2010 A time-resolved investigation of the Hall thruster breathing mode Ph.D. thesis University of Michigan

[22] Touzeau M, Prioul M, Roche S, Gascon N, Pérot C, Darnon F, Béchu S, Philippe-Kadlec C, Magne L, Lasgorceix P et al. 2000 Plasma Physics and Controlled Fusion 42 B323

[23] Bouchoule A, Philippe-Kadlec C, Prioul M, Darnon F, Lyszyk M, Magne L, Pagnon D, Roche S, Touzeau M, Béchu S et al. 2001 Plasma Sources Science and Technology 10364

[24] Grimaud L, Pétin A, Vaudolon J and Mazouffre S 2016 Review of Scientific/Instruments 87043506

[25] Nakles M R, Holmes M R and Hargus Jr W A 2015 An investigation into the spectral imaging of Hall thruster plumes Proceedings of the 34th International Electric Propulsion Conference IEPC-2015-416 (Hyogo-Kobe, Japan)

[26] Liu D, Huffman R E, Branam R D and Hargus, Jr W A 2011,IEEE Transactions on Plasma Science 39 2926-2927

[27] Diallo A, Keller S, Shi Y, Raitses Y and Mázouffre S 2015 Review of Scientific Instruments 86 033506

[28] Mazouffre S and Bourgeois G 2010 Plasma Sources Science and Technology 19065018

[29] Durot C J, Georgin M P and Gallimore A D 2015 Time-resolved laser-induced fluorescence measurements in the plume of a 6-kW Hall thruster Proceedings of the 34th International Electric Propulsion Conference IEPC-2015-399 (Hyogo-Kobe, Japan)

[30] Lucca Fabris A, Young C V and Cappelli M A 2015 Journal of Applied Physics 118233301

[31] Romadanov I, Raitses Y, Diallo A, Hara K, Kaganovich I and Smolyakov A 2018 Physics of Plasmas 25033501

[32] Karabadzhak G F, Chiu Y h and Dressler R A 2006 Journal of Applied Physics 99113305

[33] MacDonald N A, Cappelli M A and Hargus Jr W A 2012 Review of Scientific Instruments 83 113506

[34] Mazouffre S, Gawron D and Sadeghi N 2009 Physics of Plasmas 16043504

[35] Durot C, Gallimore A and Smith T 2014 Review of Scientific Instruments 85013508

[36] Lucca Fabris A, Young C V and Cappelli M A 2015 Time-synchronized laser induced fluorescence techniques for the study of quasi-periodic xenon plasma phenomena Proceedings of the 34th International Electric Propulsion Conference IEPC-2015-349 (Hyogo-Kobe, Japan)

[37] Liebeskind J G, Hanson R K and Cappelli M A 1991 Lif measurements of species velocities in an arcjet plume Proceedings of the 22nd International Electric Propulsion Conference IEPC-91-091 (Viareggio, Italy)

[38] Liebeskind J G, Hanson R K and Cappelli M A 1993 Applied Optics 32 6117-6127

[39] Pobst J, Wysong I and Spores R 1995 Laser induced fluorescence of ground state hydrogen atoms at nozzle exit of an arcjet thruster 26th Plasmadynamics and Lasers Conference AIAA Paper 95-1973 (San Diego, CA) p 1973

[40] Storm P V and Cappelli M A 1996 AIAA Journal 34 853-855

[41] Storm P V and Cappelli M A 1996 Applied Optics 35 4913-4918

[42] Storm P V and Cappelli M A 1998 Applied Optics 37 486-495

[43] Walker Q, Hargus W and Cappelli M 1998 Characterization of a low-power helium arcjet 
34th AIAA/ASME/SAE/ASEE Joint Propulsion Conference and Exhibit AIAA Paper 98-3636 (Cleveland, OH) p 3636

[44] Manzella D H 1994 Stationary plasma thruster ion velocity distribution Proceedings of the 30th AIAA/ASME/SAE/ASEE Joint Propulsion Conference \& Exhibit AIAA-1994-3141 (Indianapolis, IN)

[45] Cedolin R, Hanson R and Cappelli M 1994 Semiconductor laser diagnostics for xenon plasmas 30th Joint Propulsion Conference and Exhibit AIAA Paper 94-2739 (Indianapolis, IN) p 2739

[46] Cedolin R J, Hargus, Jr W A, Storm P V, Hanson R K and Cappelli M A 1997 Applied Physics B 65 459-469

[47] Hargus Jr W and Cappelli M 1998 Laser induced fluorescence measurements on a laboratory hall thruster 34th AIAA/ASME/SAE/ASEE Joint Propulsion Conference and Exhibit AIAA Paper 98-3645 (Cleveland, OH) p 3645

[48] Williams Jr G, Gallimore A, Smith T, Gulczinski III F, Beal B and Drake R 1999 Laser induced fluorescence measurement of ion velocities in the plume of a hall effect thruster 35th Joint Propulsion Conference and Exhibit AIAA Paper 99-2424 (June 20-24)

[49] Smith T B, Hermanz D A, Gallimore A D and Drake R P 2001 Proceedings of the 27th Ienternationl Electric Propulsion Conference IEPC-01-0019

[50] Smith T, Ngom B, Linnell J and Gallimore A 2005 Diode laser-induced fluorescence of xenon ion velocity distributions 41st AIAA/ASME/SAE/ASEE Joint Propulsion Conference E Exhibit AIAA Paper 2005-4406 (Tucson, AZ) p 4406

[51] Dorval N, Bonnet J, Marque J, Rosencher E, Chable S, Rogier F and Lasgorceix P 2002 Journal of Applied Physics 91 4811-4817

[52] Huang W, Smith T B and Gallimore A D 2009 Obtaining velocity distribution using a xenon ion line with unknown hyperfine constants 40th AIAA Plasmadynamics and Laser Conference AIAA-2009-4226 (San Antonio, TX)

[53] Gawron D, Mazouffre S, Sadeghi N and Héron A 2008 Plasma Sources Science and Technology 17 025001

[54] Hargus Jr W A and Charles C S 2010 Journal of Propulsion and Power 26 135-141

[55] Beiting E J, Spektor R, Diamant K D, Corey R and Delgado J 2013 Near field maps of xenon ion velocity of the spt-140 hall thruster by laser induced fluorescence Proceedings of the 33rd International Electric Propulsion Conference IEPC-2013-053 (Washington, DC)

[56] MacDonald N A, Cappelli M A and Hargus Jr W A 2014 Journal of Physics D: Applied Physics 47115204

[57] Young C V, Lucca Fabris A and Cappelli M A 2015 Applied Physics Letters 106044102

[58] Demtröder W 1996 Laser spectroscopy: Basic concepts and instrumentation 2nd ed (Springer, Berlin Heidelberg New York)

[59] Hanson R K, Spearrin R M and Goldenstein C S 2016 Spectroscopy and optical diagnostics for gases (Springer International Publishing Switzerland)

[60] Young C V 2016 Dynamics of Plasma Discharges Used for Space Propulsion Ph.D. thesis Stanford University

[61] Biloiu C, Sun X, Choueiri E, Doss F, Scime E, Heard J, Spektor R and Ventura D 2005 Plasma Sources Science and Technology 14766

[62] Biloiu I A, Sun X and Scime E E 2006 Review of Scientific Instruments 77 10F301

[63] Desecures M, de Poucques L, Easwarakhanthan T and Bougdira J 2014 Applied Physics Letters 105181120

[64] Vaudolon J, Balika L and Mazouffre S 2013 Review of Scientific Instruments 84073512

[65] Diallo A, Shi Y, Keller S, Raitses Y and Mazouffre S 2013 Time-dependent ion velocity distribution: A novel heterodyne laser-induced fluorescence with coupled wave excitation Proceedings of the 33rd International Electric Propulsion Conference IEPC-2013-239 (The George Washington University, Washington, D.C.)

[66] Keller S, Raitses Y and Diallo A 2014 Driving low frequency breathing oscillations in a Hall thruster 
50th AIAA/ASME/SAE/ASEE Joint Propulsion Conference AIAA 2014-3509 (Cleveland, OH)

[67] Durot C J, Gallimore A D and Smith T B 2013 Development and validation of a novel time-resolved laser-induced fluorescence technique Proceedings of the 33rd International Electric Propulsion Conference IEPC-2013-356 (The George Washington University, Washington, D.C.)

[68] Durot C J 2016 Development of a Time-Resolved Laser-Induced Fluorescence Technique for Nonperiodic Oscillations Ph.D. thesis University of Michigan

[69] MacDonald N A 2012 Laser induced fluorescence characterization of cusped field plasma thrusters Ph.D. thesis Stanford University

[70] Lucca Fabris A, Young C V and Cappelli M A 2015 Plasma Sources Science and Technology 24 055013

[71] Raitses Y and Fisch N 2001 Physics of Plasmas 8 2579-2586

[72] Smith A W 2010 Field structure and electron transport in the near-field of coaxial Hall thrusters Ph.D. thesis Stanford University

[73] Busek Company Inc 2016 BHT-600 Busek Hall effect thruster datasheet, [Online]. Available: http: //www. busek.com/index_htm_files/70000701\%20BHT-600\%20Data\%20Sheet\%20Rev-.pdf. [2018, Apr 22]. Natick, MA.

[74] Ekholm J M, Hargus Jr W A, Larson C W, Nakles M/R, Reed G D and Niemela C S 2006 Plume characteristics of the Busek $600 \mathrm{~W}$ Hall thruster Proceedings of the 42nd AIAA/ASME/SAE/ASEE Joint Propulsion Conference and Exhibit AIAA-2006-4659 (Sacramento, CA)

[75] Victor A L, Zurbuchen T and Gallimore A D 2006 Journal of Propulsion and Power 22 1421-1424

[76] Gonzales A E, Koo J W and Hargus, Jr W A 2014 IEEE Transactions on Plasma Science 42 806-812

[77] Hargus Jr W A and Charles C S 2008 Journal of Propulsion and Power 24 127-133

[78] Bareilles J, Hagelaar G J M, Garrigues L, Boniface C, Boeuf J P and Gascon N 2004 Physics of Plasmas 11 3035-3046

[79] Hara K, Sekerak M J, Boyd I D and Gallimore A D 2014 Journal of Applied Physics 115203304

[80] Yang L, Zhang T, Chen J and Jia Y 2018 Plasma Science and Technology 20075503

[81] Scharfe M K, Gascon N, Cappelli M A and Fernandez E 2006 Physics of Plasmas 13083505

[82] Parra F, Ahedo E, Fife J and Martinez-Sanchez M 2006 Journal of Applied Physics 100023304

[83] Nakles M R and Hargus Jr W A 2011 Journal of Propulsion and Power 27 737-743

[84] Pérez-Luna J, Hagelaar G J M, Garrigues L and Boeuf J P 2009 Plasma Sources Science and Technology 18034008 